\title{
Secrétariat de la formation prégraduée, postgraduée et continue (FPPC): rapport d'activité 2005
}

Christoph Hänggeli

* Loi fédérale concernant l'exercice des professions de médecin, de pharmacien et de vétérinaire dans la Confédération suisse.

Correspondance:

Christoph Hänggeli, avocat FMH

Elfenstrasse 18

CH-3000 Berne 15

ch@fmh.ch

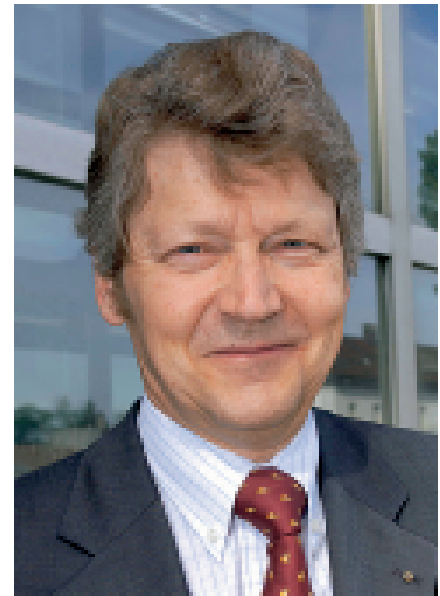

Dr Max Giger,

responsable du domaine

de la formation médicale

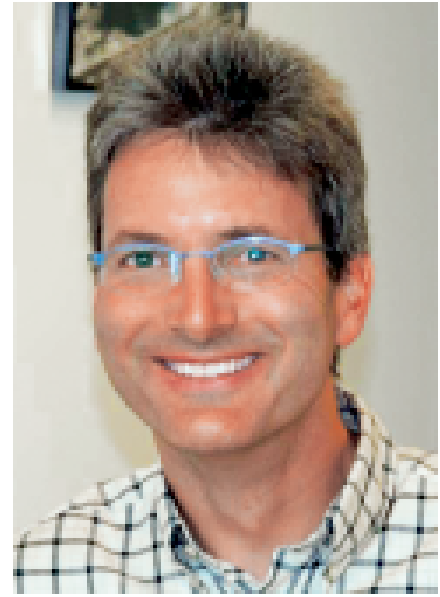

Christoph Hänggeli, avocat, administrateur responsable du secrétariat de la FPPC

\section{Tâches et objectifs: le secrétariat FPPC comme centre de services de la FMH}

Le secrétariat FPPC est au service des médecins, des institutions et des autorités pour toutes les questions concernant la formation postgraduée et continue des médecins. Nos tâches principales portent sur tout ce qui concerne l'octroi de titres de formation postgraduée et la reconnaissance des établissements de formation postgraduée, avec pour objectif premier de fournir un service compétent aux médecins souhaitant obtenir un titre fédéral de spécialiste ou un autre titre. En outre, notre secrétariat assiste tous les organes et commissions œuvrant dans le domaine de la formation postgraduée et continue. Il soutient notamment les sociétés de discipline médicale et d'autres organisations dans l'accomplissement de leurs tâches, telles que l'élaboration et la révision des programmes de formation postgraduée.

Le secrétariat FPPC exerce son activité sur la base et dans le cadre de la loi sur l'exercice des professions médicales (LEPM)*, laquelle confie depuis 2002 la haute surveillance de la formation postgraduée médicale à la Confédération. La FMH est responsable de l'application concrète des dispositions légales et s'appuie, pour ce faire, sur la Réglementation pour la formation postgra- duée et les 44 programmes de formation postgraduée accrédités. La réalisation de cette tâche gouvernementale par une association privée nécessite une délimitation claire avec les autres activités de la FMH. Par conséquent, le secrétariat FPPC est un département indépendant sur le plan administratif et forme un centre de services distinct au niveau financier. Toutes les charges et les recettes font l'objet d'une comptabilité séparée, ce qui permet d'assurer la transparence d'un domaine essentiellement financé par des taxes.

Quels objectifs concrets avons-nous atteints durant l'exercice écoulé? Mentionnons tout d'abord deux projets achevés avec succès (cf. aussi ch. 3):

- Le 31 mai 2005, le Département fédéral de l'intérieur (DFI) a accrédité les 44 programmes de formation postgraduée pour sept nouvelles années. Les efforts importants accomplis par la FMH et ses sociétés de discipline médicale sous la pression du temps ont été récompensés. La décision d'accréditation est liée à l'obligation d'introduire l'éthique et l'économie de la santé dans tous les programmes de formation postgraduée. 
- L'optimisation des prestations et processus internes du secrétariat FPPC s'est poursuivie. Une enquête réalisée durant l'exercice écoulé auprès des clients confirme le haut degré d'attention que leur portent nos collaboratrices et collaborateurs. Les besoins des médecins en formation postgraduée doivent rester le critère essentiel pour l'élaboration et la qualité des prestations de services du secrétariat FPPC.

Tâches générales du secrétariat CFPC/formation continue/site internet

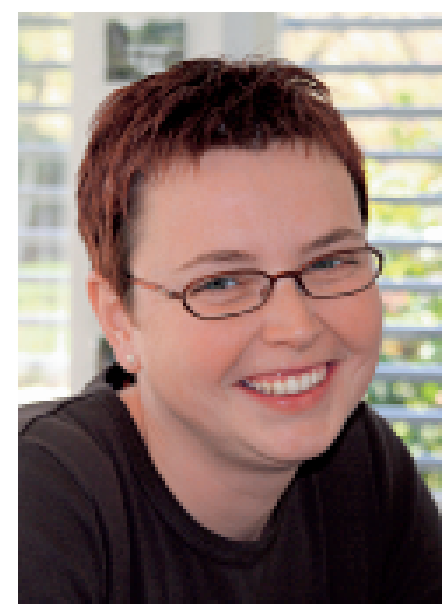

Petra Baeriswyl

\section{Ressources humaines}

Pour les questions professionnelles, le secrétariat FPPC est placé sous la direction du Dr Max Giger, membre du Comité central et responsable du domaine de la formation médicale, qui simultanément préside, ex officio et en union de personnel, la Commission pour la formation postgraduée et continue (CFPC), la Commission des titres (CT) et la Commission des établissements de formation postgraduée (CEFP).

Le secrétariat FPPC s'articule en trois domaines:

Etablissements de formation postgraduée/ Commission des établissements de formation postgraduée (CEFP)

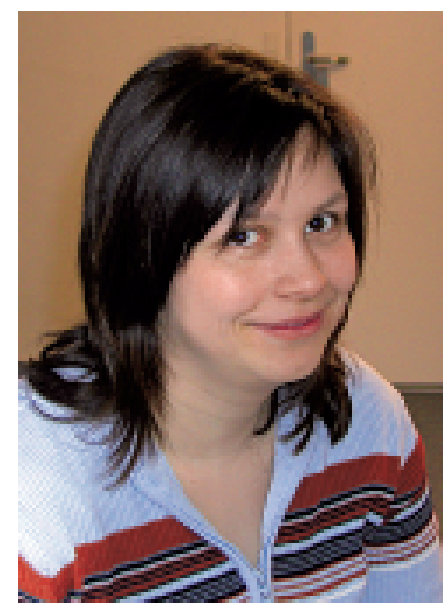

Renate Jungo

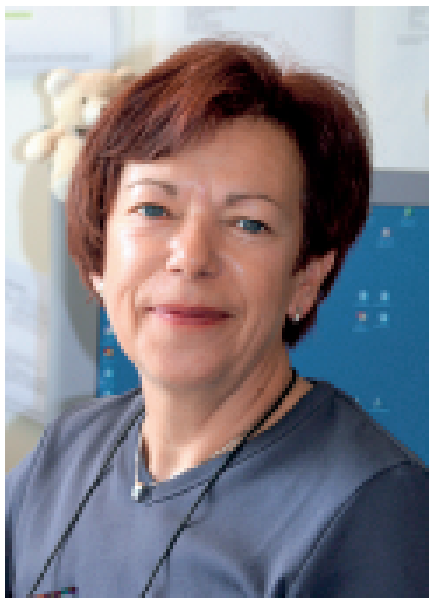

Esther Würz (responsable d'équipe)

Diplômes/Commission des titres (CT)

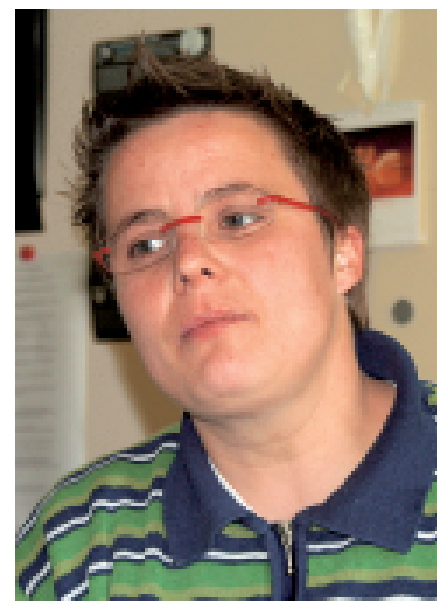

Katrin Flück

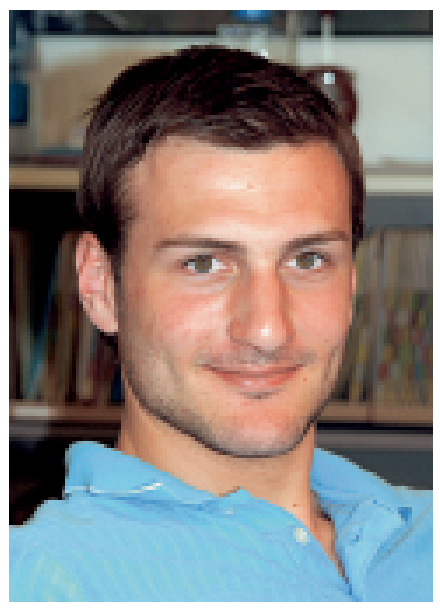

Severin Gebhart

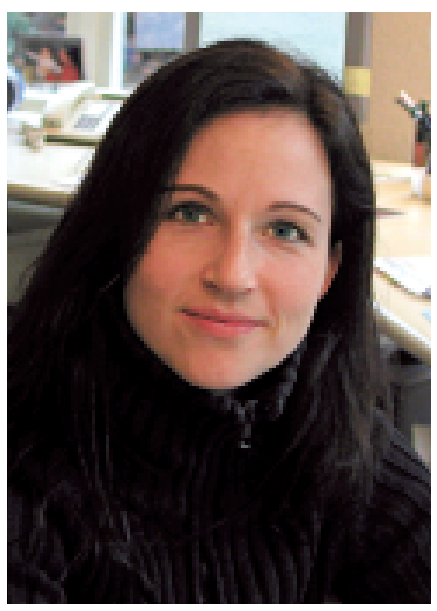

Caroline Gomez Hdz. 


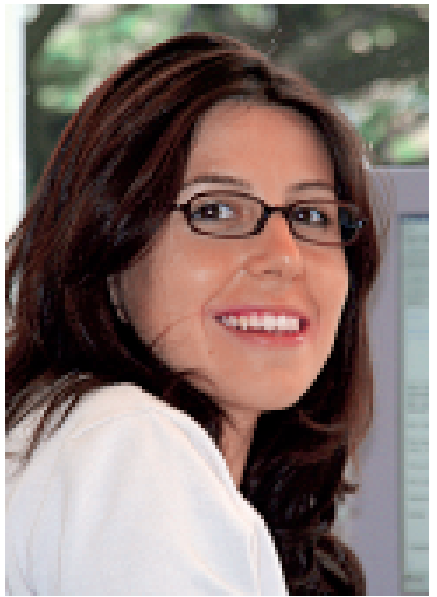

Carmela Hostettler

\section{Diplômes/Commission des titres (CT)}

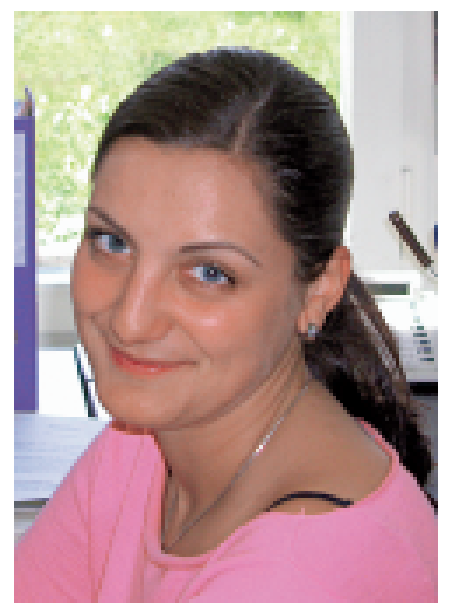

Jasmine In-Albon

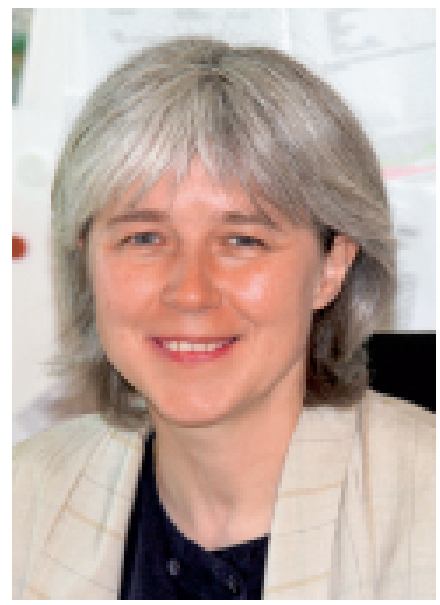

Simone Minder

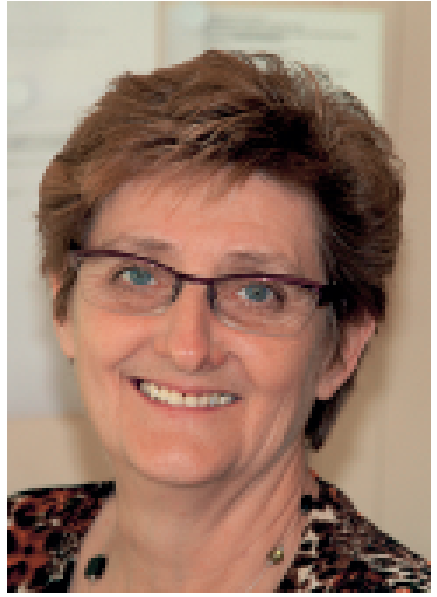

Esther Rüegg

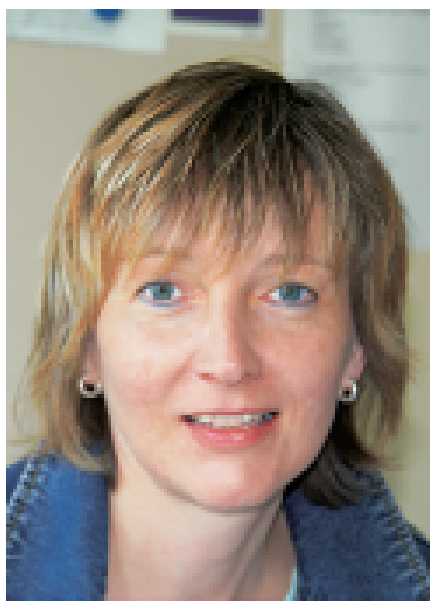

Therese von Dach

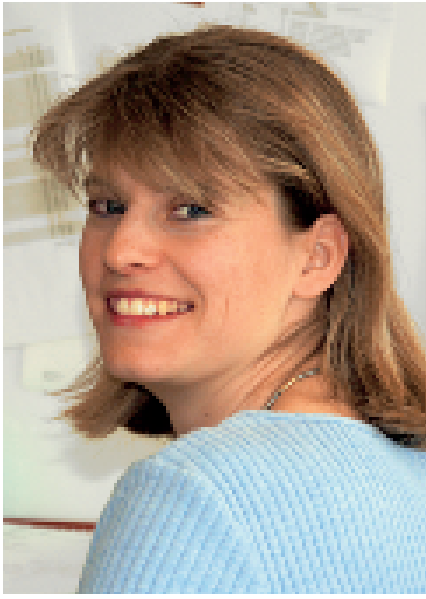

Julia Schaad www.fmh.ch/awf $\rightarrow$ Divers $\rightarrow$ Le Secrétariat FPPC se présente
Le secrétariat FPPC peut en outre profiter de tous les services du Secrétariat général (service de traduction, service juridique, informatique, comptabilité, administration des membres, etc.). Grâce à une comptabilité analytique efficace et des décomptes internes, la transparence des coûts pour toutes les tâches principales est garantie en tout temps.

\section{5 sous la loupe: quel est votre degré de satisfaction quant aux prestations de service du secrétariat FPPC?}

Pourquoi une enquête auprès de la clientèle? $\mathrm{Au} 1^{\mathrm{er}}$ juin 2005, autrement dit à la date d'expiration de l'accréditation spéciale, le Département fédéral de l'intérieur (DFI) a décidé de la renouveler pour les 44 programmes de formation postgraduée de la FMH pour une durée de sept ans. Par conséquent, la FMH reste la principale responsable de la réglementation et de l'organisation de la formation postgraduée des médecins en Suisse. Après chaque période d'accréditation, il est possible de mandater une autre organisation nationale. Cette exigence permet de garantir que la FMH s'adapte continuellement aux nouvelles exigences et développements en la matière car si tel ne devait pas être le cas, alors ses jours en tant que garante de la formation postgraduée des médecins seraient comptés. Dans ce contexte, il est impératif de fournir des prestations optimales à tous les médecins qui souhaitent acquérir un titre postgrade fédéral. C'est notamment dans ce but que le Secrétariat de la formation prégraduée, postgraduée et continue (FPPC) qui, de concert avec les organes de décision compétents, œuvre en tant que centre de services pour la mise en vigueur et l'application 
de la Réglementation pour la formation postgraduée, a effectué durant l'exercice écoulé une enquête auprès des médecins, ses «clients».

Parmi les services fournis par le secrétariat FPPC, les conseils personnalisés, les réponses à des questions, l'établissement de plans de formation postgraduée ainsi que le traitement des demandes de titres jouent un rôle prépondérant. Une autre tâche importante concerne l'élaboration des programmes de formation postgraduée. L'enquête a porté sur tous les médecins ayant obtenu un titre de spécialiste entre le $1^{\mathrm{er}}$ avril et le $1^{\text {er }}$ juillet 2005 . Avec 224 questionnaires envoyés, dont 138 nous sont parvenus en retour, le taux de réponse s'est monté à $62 \%$, ce qui est extraordinairement élevé pour une enquête effectuée sans rappel.

\section{Résultats de l'enquête}

Cette enquête a fourni de précieuses informations quant aux points positifs et négatifs des prestations fournies par le secrétariat FPPC. Les critiques concernant les différents programmes de formation postgraduée se sont avérées particulièrement instructives. Les points suivants feront ici l'objet d'une mention spéciale:

\section{Formulaire de demande en ligne; utilisa-} tion et fonctionnalités du site internet Le site internet du secrétariat FPPC fait office de plaque tournante pour toutes les informations et tous les contacts se rapportant à la formation postgraduée et continue. Tout médecin qui souhaite poser une question, déposer une demande de titre ou établir un plan de formation peut utiliser le formulaire électronique en ligne prévu à cet effet. Les résultats de l'enquête montrent cependant que malgré des adaptations continuelles, ce formulaire doit encore être amélioré. Dans l'ensemble, un cinquième des médecins interrogés n'a pas été en mesure d'effectuer une demande de titre en ligne et le même nombre n'a pas trouvé toutes les informations souhaitées sur le site. Là aussi, des mesures d'amélioration s'imposent.

\section{Compétences et amabilité \\ des collaborateurs (fig. 1 et 2)}

A ce propos, nous avons cherché à savoir si nos «clients» se sentaient soutenus par nos collaborateurs et s'ils les jugeaient aimables et compétents. Au vu des résultats, tant les compétences que l'amabilité et la serviabilité des collaborateurs du secrétariat FPPC ont été jugées de manière extrêmement positive. Sur une échelle allant de 1 à 5, $75 \%$ et $87 \%$ des sondés ont donné la note maximale à ces deux caractéristiques respectives, signe de satisfaction en ce qui concerne la procédure d'attribution des titres.

Temps de traitement des demandes (fig. 3) Le temps nécessaire au traitement des dossiers est un élément-clé en matière de satisfaction de la clientèle. La moitié de tous les médecins qui ont déposé une demande de titre estiment que le temps consacré au traitement de cette dernière est très court. En outre, $41 \%$ des sondés le jugent satisfaisant et $7 \%$ l'estiment trop long. En réalité, la moitié des demandes de titre a été traitée dans les 3 semaines. Il s'agit d'une très bonne moyenne comparée à ce qui se faisait auparavant. Cependant, et grâce au traitement accru

Figure 1

Comment jugez-vous les compétences des collaborateurs du secrétariat FPPC qui vous ont renseigné?

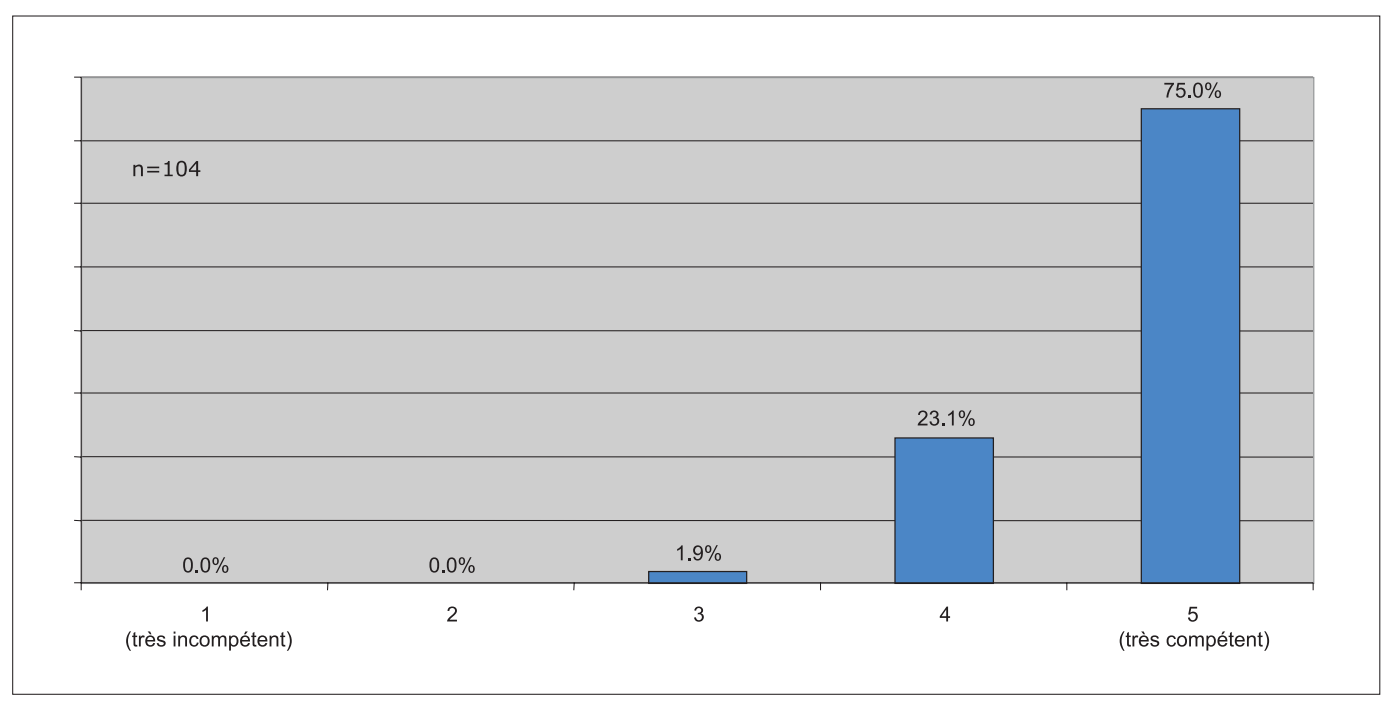


Figure 2

Comment évaluez-vous l'amabilité/serviabilité des collaborateurs du secrétariat FPPC qui vous ont renseigné?

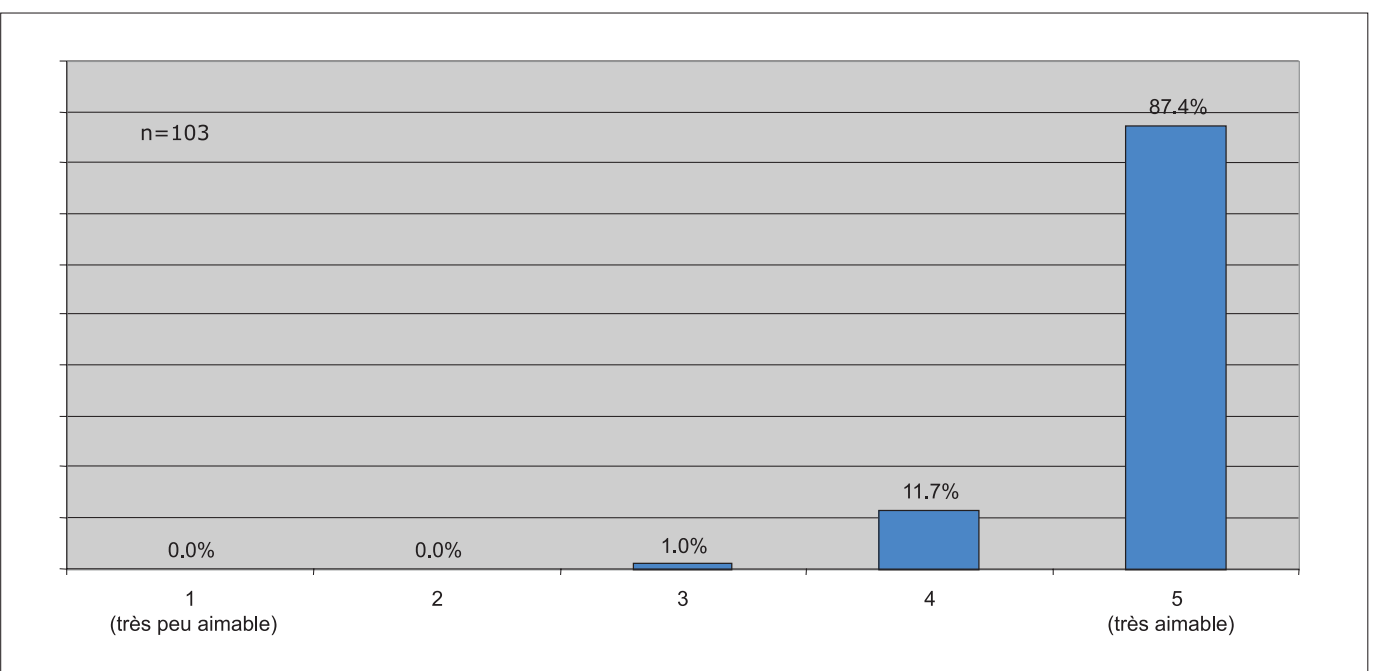

Figure 3

Comment jugez-vous le temps consacré au traitement de votre demande?

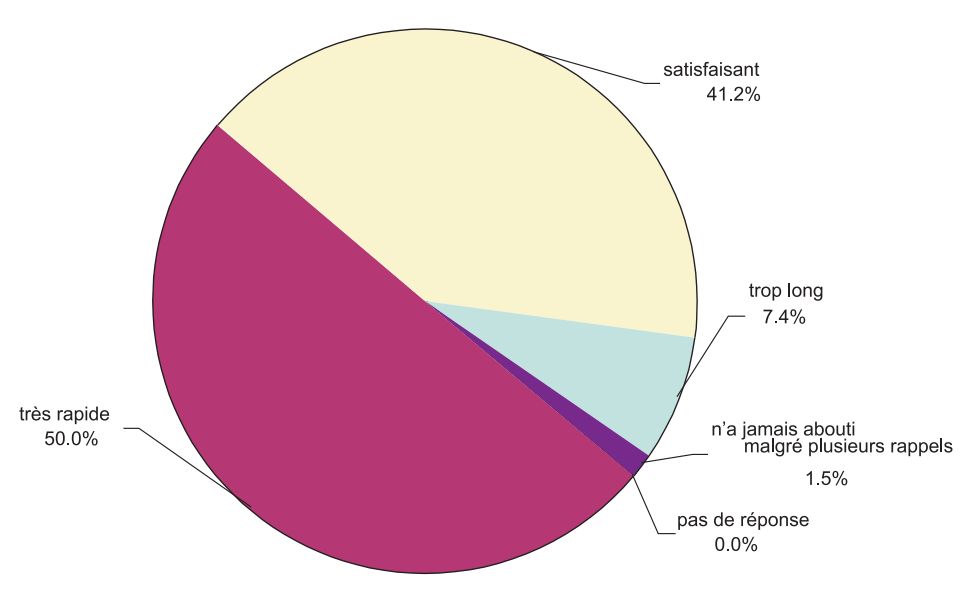

Figure 4

Les règles stipulées par votre programme de formation postgraduée sont-elles claires et compréhensibles?

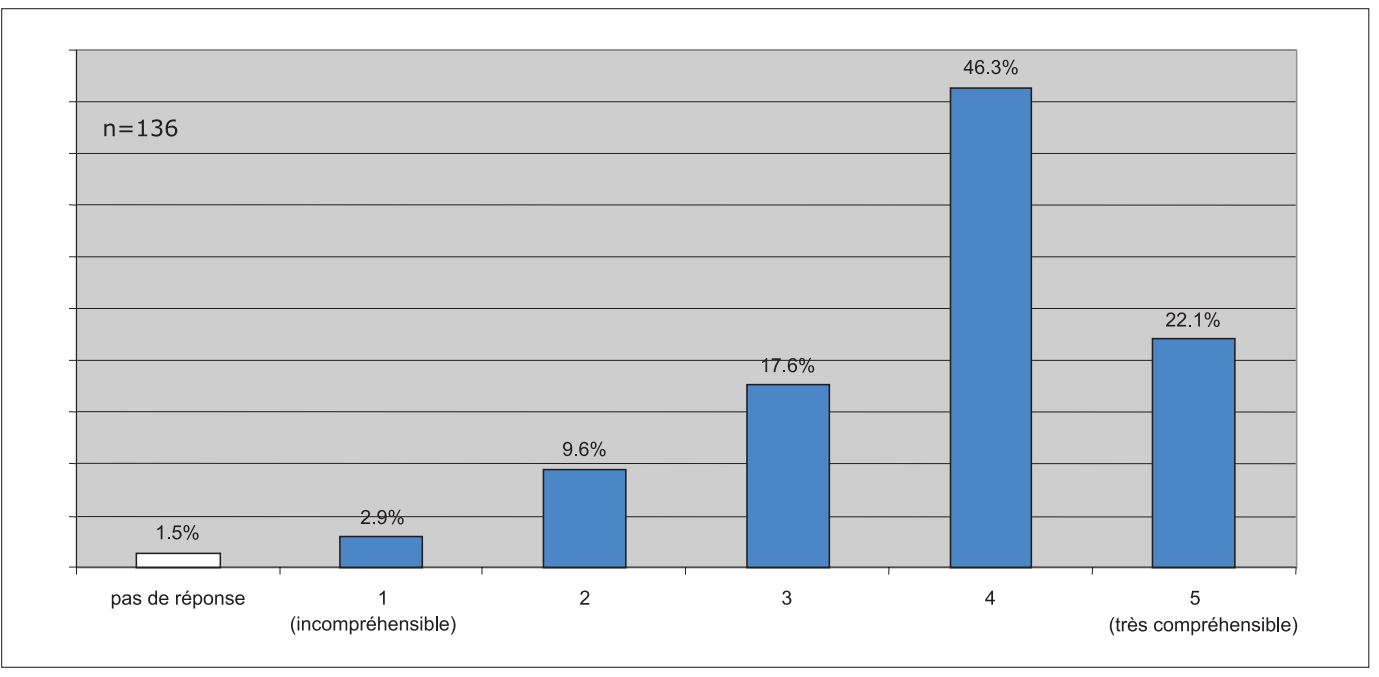


des dossiers par voie électronique, il sera possible d'améliorer encore la rapidité de la procédure.

\section{Satisfaction quant aux programmes} de formation postgraduée (fig. 4)

Environ 70\% des médecins interrogés considèrent que leur programme de formation postgraduée est clair et compréhensible (notes 4 et 5). Ce point de vue diffère cependant d'une discipline médicale à l'autre. Le programme de formation en psychiatrie et psychothérapie a notamment fait l'objet de nombreuses critiques. Les points mis en exergue devront être discutés avec les sociétés de discipline médicale concernées. Les énoncés à améliorer concernent les dispositions transitoires - ce en raison des multiples révisions auxquelles sont soumis les programmes de formation - et les examens de spécialiste. En outre, il est également impératif de discuter du niveau des exigences de formation postgraduée en Suisse en comparaison européenne. A ce propos, il est intéressant de constater que des demandes de révision émanant des sociétés de discipline médicale elles-mêmes ont d'ores et déjà été déposées en vue d'une réduction des exigences en matière de formation postgraduée.

\section{Autres informations}

La présente enquête, effectuée et évaluée dans le cadre d'un mémoire de master, peut être consultée dans son intégralité, mais en allemand seulement, sur le site internet de la FMH.

$$
\text { www.fmh.ch/ww/fr/pub/awf.htm } \rightarrow \text { Thèmes d'actua- }
$$
lité

La qualité de la formation postgraduée dans les cliniques et les hôpitaux fait, quant à elle et depuis de nombreuses années, l'objet d'une enquête séparée qui peut également être consultée en ligne.

www.fmh.ch/ww/fr/pub/awf.htm $\rightarrow$ Formation postgraduée $\rightarrow$ Enquête sur la qualité de la formation postgraduée

\section{Diplômes/Commission des titres (CT)}

Après le flot de demandes sans précédent observé en 2002 et 2003 à la suite du blocage de l'accès à la pratique privée et de l'entrée en vigueur des accords bilatéraux, le nombre de titres décernés en 2004 et 2005 s'est stabilisé. Néanmoins, le chiffre de 1039 titres de spécialiste se situe encore bien au-dessus de la moyenne enregistrée pendant des années et il est impossible de savoir si l'on redescendra un jour aux chiffres antérieurs de 800 à 900 titres par année (tab 1 et fig. 5). Le fait que le nombre de demandes reste aussi élevé ne s'explique plus par le blocage de l'accès à la pratique privée. Il est plutôt dû au concept de la valeur intrinsèque selon le TARMED. Tous les titres de formation postgraduée susceptibles d'être obtenus sont demandés, afin d'éviter de ne pouvoir facturer certaines positions faute de «valeur intrinsèque» dûment recensée.

La durée de traitement des demandes varie très fortement d'une spécialité à l'autre. En moyenne, la moitié de toutes les demandes a pu être réglée en l'espace de 25 jours (fig. 6).

Outre les demandes de titre, les collaboratrices et collaborateurs du secrétariat FPPC établissent des plans de formation postgraduée donnant des renseignements juridiquement contraignants sur les conditions déjà remplies par rapport à un programme de formation postgraduée. Au cours de l'exercice écoulé, le secrétariat FPPC a établi 659 plans de formation postgraduée au total en collaboration avec la Commission des titres.

$w w w . f m h . c h / a w f$

\section{Etablissements de formation postgraduée et Commission des établissements de formation postgraduée (CEFP): recours à trois piliers pour améliorer la qualité de la formation}

\section{Généralités}

La reconnaissance et la classification des cliniques et des cabinets médicaux incombent à la Commission des établissements de formation

\begin{tabular}{|c|c|c|c|c|}
\hline & Total & Titres de spécialiste & Formations approfondies & Médecins praticiens \\
\hline 2002 & 2201 & 1609 & 119 & 473 \\
\hline 2003 & 2277 & 1551 & 472 & 254 \\
\hline 2004 & 1579 & 1140 & 319 & 120 \\
\hline 2005 & 1360 & 1039 & 186 & 135 \\
\hline
\end{tabular}


Figure 5

Diplômes décernés entre 1996 et 2005 (comparaison sur dix ans).

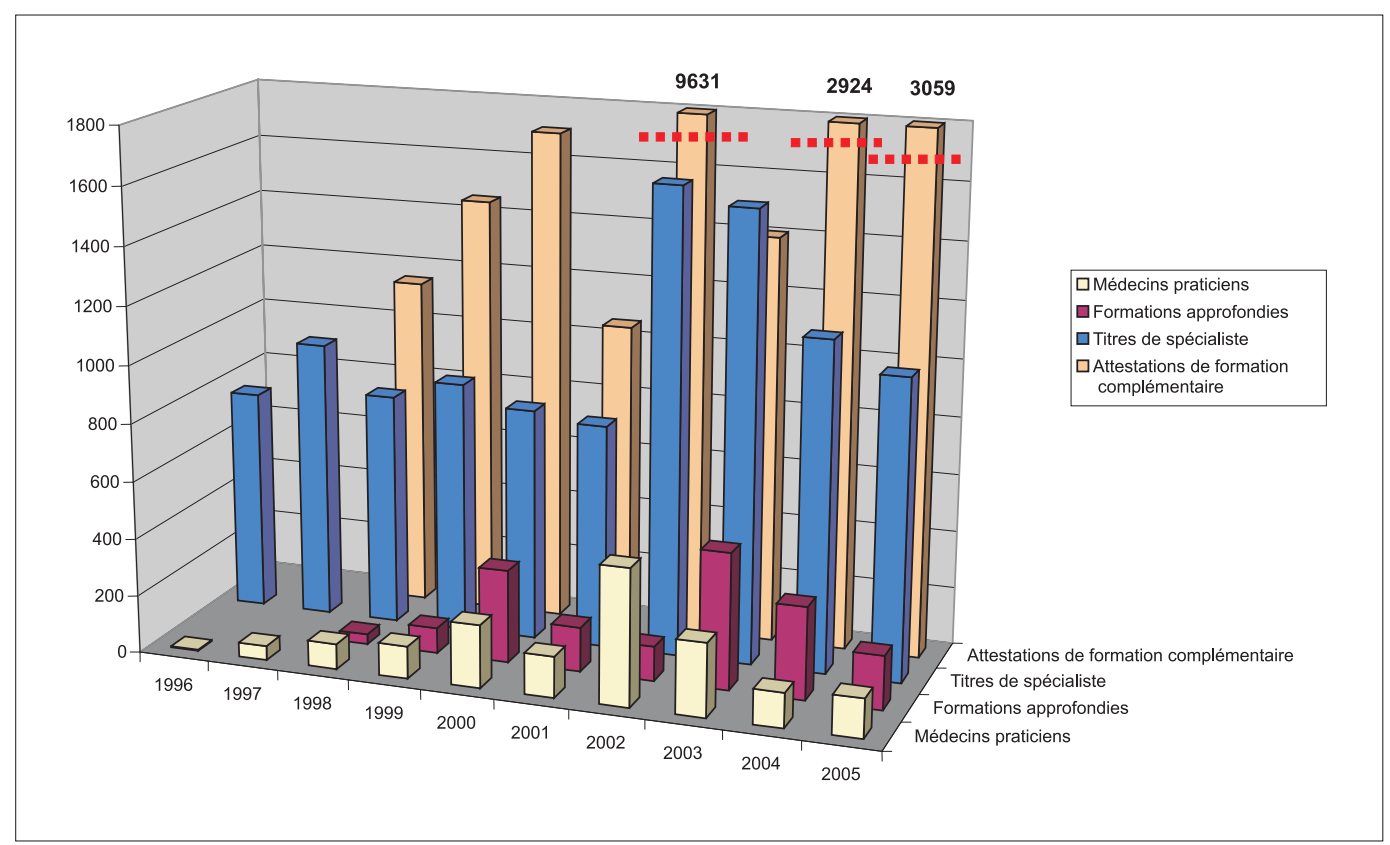

Figure 6

Durée de traitement des demandes par spécialité.

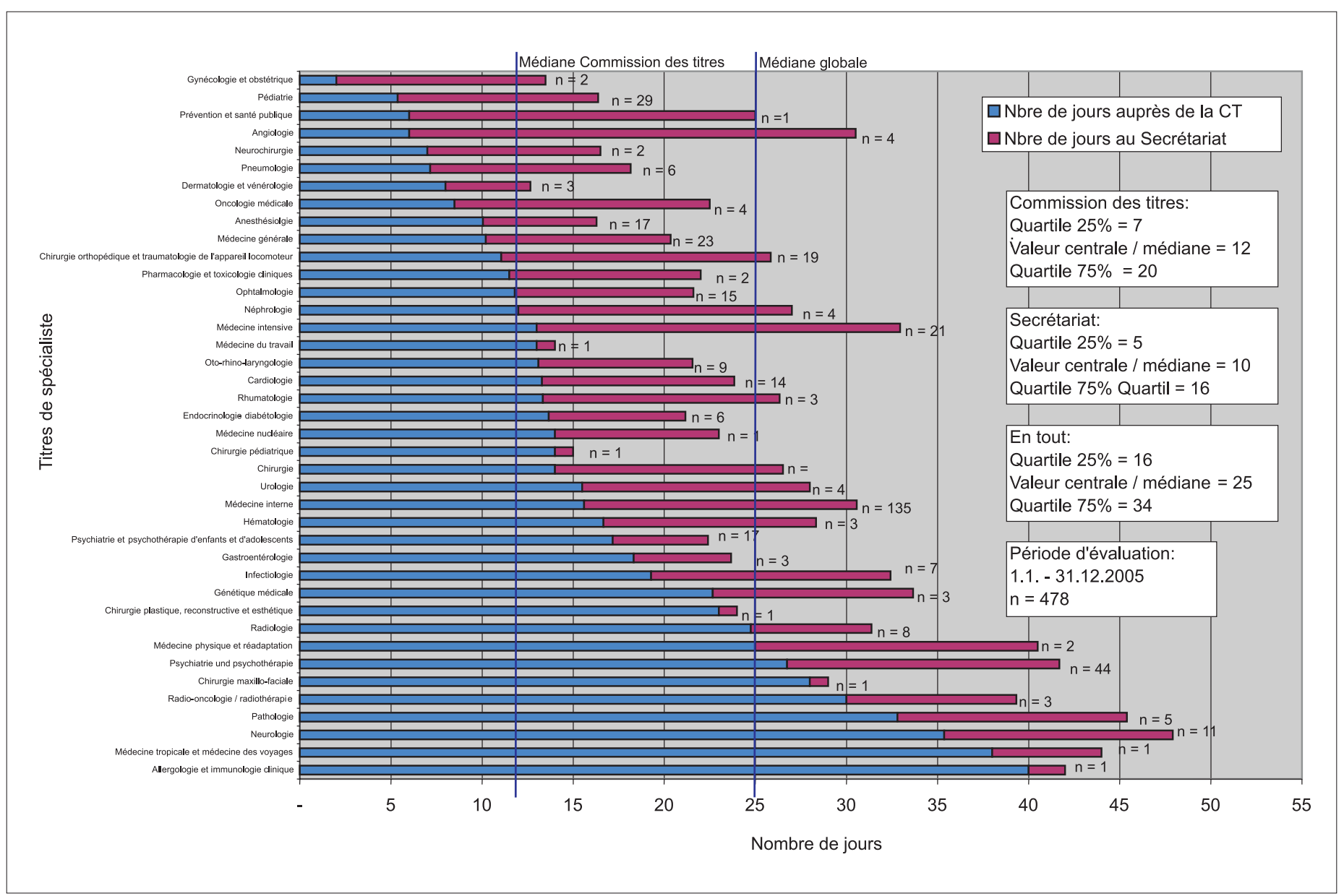


postgraduée (CEFP), constituée d'un délégué membre de la société de discipline médicale concernée et d'un délégué étranger à la discipline.

Lorsque les critères de reconnaissance changent dans le cadre d'un programme de formation postgraduée, tous les établissements de la discipline concernée doivent être réévalués. Il en va de même lors d'un changement de responsable ou de médecin-chef.

$\mathrm{Au}$ cours de l'exercice passé sous revue, la Commission des établissements de formation postgraduée (CEFP) a

- reconnu 104 nouveaux établissements (dont 56 cabinets médicaux),

- réévalué 101 établissements (confirmations et nouvelles classifications),

- rayé 30 établissements (dont 6 cabinets médicaux) de la liste des établissements et cabinets médicaux de formation postgraduée reconnus et

- rejeté 17 demandes de reconnaissance comme établissement de formation postgraduée (dont 6 émanant de cabinets médicaux).

www.fmh.ch/awf $\rightarrow$ Formation postgraduée $\rightarrow$ Pour les assistants/les médecins en formation postgraduée $\rightarrow$ Liste des établissements de formation postgraduée/Cabinets médicaux

\section{Concepts de formation postgraduée} des établissements de formation postgraduée ( $1^{\text {er }}$ pilier)

Toutes les sociétés de discipline médicale ont élaboré un schéma pour les établissements de formation postgraduée reconnus dans leur domaine spécifique, schéma servant de modèle pour l'établissement d'un concept de formation postgraduée. Ce concept (ou «déclaration de qualité») doit présenter les matières enseignées du programme de formation postgraduée concerné, structurées d'après le calendrier et le contenu. A fin 2005, certains établissements de formation postgraduée ne disposaient toujours pas d'un concept (fig. 7). Ces concepts peuvent s'avérer très utiles aux médecins-assistants pour choisir leur prochain poste de formation postgraduée.

www.fmh.ch/awf $\rightarrow$ Pour les médecins-chefs/formateurs $\rightarrow$ Concepts de formation postgraduée

\section{Visites d'établissement ( $2^{\mathrm{e}}$ pilier)}

Parmi les mesures prises pour assurer la qualité de la formation postgraduée, les visites d'établissement sont le deuxième pilier porteur après les concepts de formation postgraduée. Une équipe de trois personnes, composée d'un représentant

Figure 7

Disciplines disposant de concepts de formation postgraduée dans au moins $50 \%$ des cas.

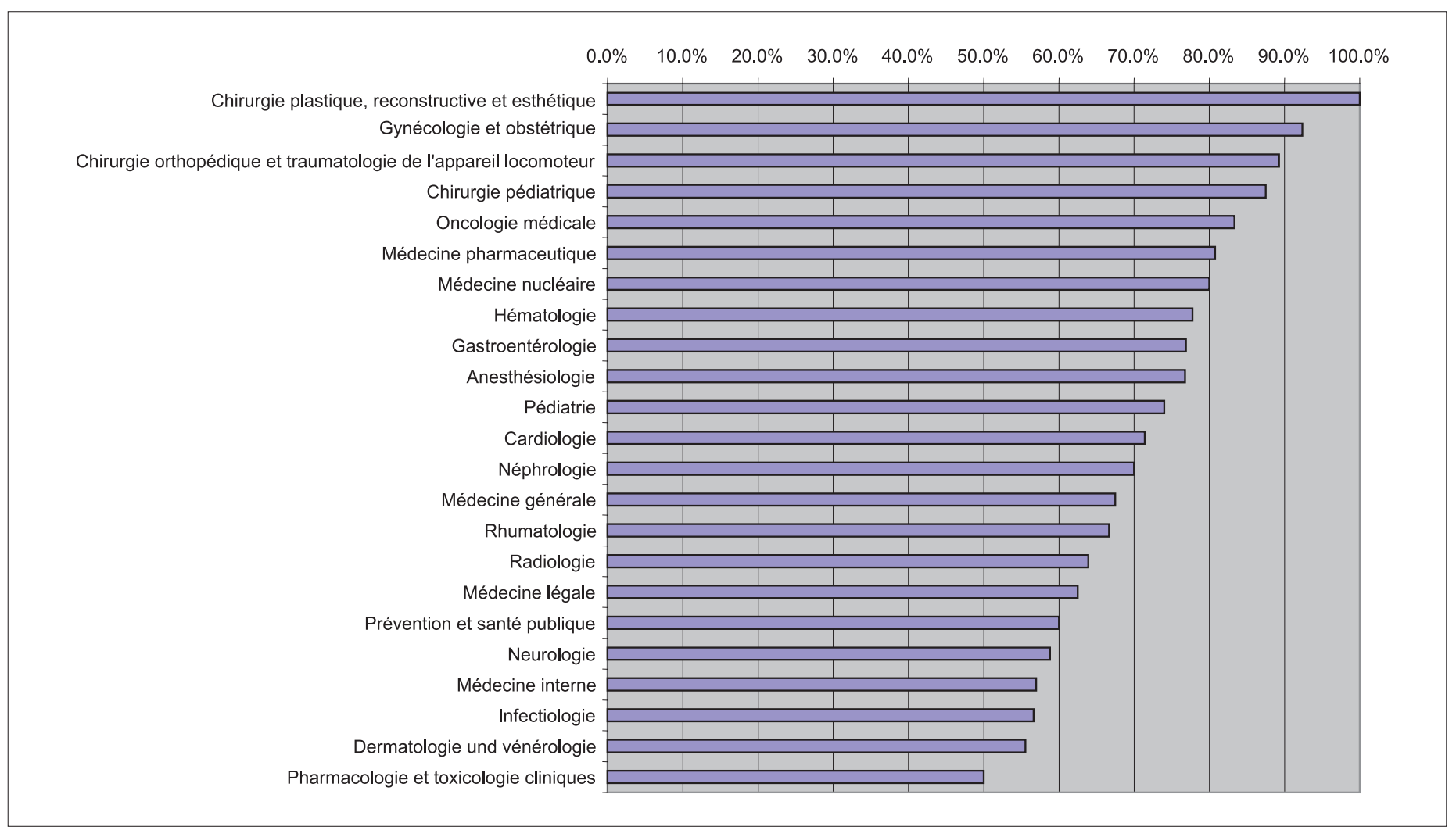


de la discipline coordonnant la visite, d'un représentant de l'ASMAC et d'un expert étranger à la discipline et désigné par la CFPC, examine sur place les conditions de formation postgraduée d'un établissement et vérifie si elles concordent avec les critères exigés pour la reconnaissance. A fin 2005, près de la moitié des sociétés de discipline médicale avaient fait usage de cet important instrument. 24 visites ont été exécutées en 2005. La Société suisse d'anesthésiologie et de réanimation évalue systématiquement les établissements de formation postgraduée et a procédé à 23 visites en quatre ans. L'équipe chargée de la visite a besoin en tout d'une journée de travail pour étudier les dossiers, se déplacer, visiter l'établissement et établir un rapport. Mais cette visite, qui exige aussi du temps et des ressources de la part de l'établissement de formation concerné, est gratifiante: les responsables d'établissement l'ont pratiquement tous considérée comme positive et bénéfique pour la formation postgraduée. Comme il s'agit d'un processus formateur, les experts peuvent discuter sur place les forces et les faiblesses du système appliqué et formuler dans leur rapport des recommandations pour les établissements visités et, le cas échéant, à l'attention de la société de discipline médicale concernée. La Commission des établissements de formation postgraduée procède ensuite à la classification définitive sur la base du rapport de visite et de la prise de position du responsable de l'établissement concerné.

Les reconnaissances et les réévaluations devraient toujours être fondées sur une visite quand les documents écrits ne suffisent pas, à eux seuls, pour une prise de décision. Dans le système de milice actuel, les sociétés de discipline médicale comme les médecins-chefs parviennent très clairement aux limites du supportable. Les sociétés de discipline médicale sont invitées à fixer des priorités et à inspecter en particulier les établissements dont la qualité de formation postgraduée est mise en doute sur la base des résultats de l'enquête.

www.fmh.ch/awf $\rightarrow$ Pour les médecins-chefs/formateurs $\rightarrow$ Visites

\section{Enquête sur la qualité de la formation postgraduée auprès des médecins-assistants ( $3^{\mathrm{e}}$ pilier)}

En 2005, l'enquête sur la qualité de la formation postgraduée, réalisée auprès des médecins-assistants, a eu lieu pour la troisième fois en collaboration avec l'Institut de psychologie sociale de l'Université de Zurich. 68\% des médecins-assistants préparant un titre de spécialiste ont répondu au questionnaire. L'évaluation des ques- tions réparties en différents domaines est d'une grande utilité, aussi bien pour les établissements de formation postgraduée que pour les formateurs concernés. Les résultats permettent en particulier de tirer des conclusions sur la qualité des processus en vigueur pour la formation postgraduée et sur les potentiels en termes d'accroissement de l'efficacité. Les résultats de l'enquête sont publiés sur le site internet.

www.fmh.ch/awf $\rightarrow$ Pour les assistants/les médecins en formation postgraduée $\rightarrow$ Enquête sur la qualité de la formation postgraduée

\section{Commission pour la formation postgraduée et continue (CFPC)}

La CFPC est l'organe consultatif du Comité central et de la Chambre médicale pour toutes les affaires concernant la formation postgraduée et continue. La CFPC est composée de délégués des sociétés de discipline médicale, des facultés de médecine, de l'ASMAC, de l'AMPHS et des associations régionales (VEDAG, SMSR, OMCT). Le plénum se réunit deux fois par année, à savoir au printemps et en automne. Les travaux préparatoires sont exécutés par un comité composé de 19 personnes. Les membres du comité assument en outre des tâches importantes en tant que délégués hors discipline au sein de la Commission des titres (CT) et de la Commission des établissements de formation postgraduée (CEFP), qui sont les deux organismes responsables des décisions concernant respectivement les titres de spécialiste et la reconnaissance des établissements de formation postgraduée. Les affaires courantes sont traitées par le bureau de la CFPC, constitué du président, le Dr Max Giger, et de deux vice-présidents, le Dr Jean-Pierre Keller et le Dr Susanna Stöhr. Durant l'année écoulée, la CFPC a traité les thèmes et les affaires suivantes avec l'appui d'intervenants éminents:

- Accréditation de la FMH

- Projet de réforme de la CFPC

- Evaluation de la formation postgraduée

- Création d'une formation approfondie en «psychiatrie et psychothérapie de la personne âgée»

- Formation continue pour la garantie des droits acquis selon le TARMED.

De surcroît, la CFPC a révisé seize programmes de formation postgraduée, huit programmes de formation complémentaire et seize programmes de formation continue.

www.fmh.ch/awf $\rightarrow$ Formation postgraduée $\rightarrow$ Bases légales $\rightarrow$ Commission pour la formation postgraduée et continue (CFPC) 


\section{Administration électronique: en route vers le bureau sans papier}

Même si l'idéal du bureau exempt de papier n'est pas encore atteint, nous travaillons d'arrachepied à l'amélioration des outils informatiques mis en place. Tous les processus sont soutenus dans la mesure du possible par les applications informatiques les plus modernes. Certains d'entre eux sont déjà entièrement automatisés. Les investissements importants réalisés dans le domaine de l'informatique nous ont permis de rationaliser les modes de fonctionnement et la structure des données et donc d'optimiser et d'accélérer nos services. En particulier:

\section{Site internet du secrétariat de la FPPC}

Le site internet du secrétariat de la FPPC s'est définitivement établi comme plaque tournante pour toutes les informations et contacts dans le domaine de la formation postgraduée et continue. Il ne sert pas seulement à la transmission classique des informations mais constitue aussi une plate-forme pour différents processus administratifs effectués électroniquement (questions, demandes de titres, reconnaissance d'établissements de formation postgraduée). Le site internet de la FPPC est un outil indispensable et un moyen de communication visité quotidiennement par 579 utilisateurs en moyenne.

www.fmh.ch/awf

\section{Formulaire de demande électronique}

Les médecins-assistants qui souhaitent poser des questions, faire établir leur plan de formation ou déposer une demande de titre utilisent le formulaire électronique ad hoc figurant sur notre site. Les demandes dûment remplies peuvent ainsi être évaluées de manière plus efficace et parviennent plus rapidement aux membres des commissions concernées. Grâce à la saisie électronique de toutes les données, il nous est possible d'établir des statistiques et des évaluations qui n'auraient pu être réalisées autrefois qu'à grand-peine et manuellement.

$w w w . f m h . c h / a w f \rightarrow$ Demandes de titre

Formulaires pour la reconnaissance des établissements de formation postgraduée Les médecins-chefs envoient eux aussi les formulaires nécessaires à la reconnaissance des établissements de formation postgraduée directement par l'internet. Tous les documents d'information de demande de reconnaissance figurent au même endroit sur notre site. www.fmh.ch/awf $\rightarrow$ Pour médecins-chefs/formateurs $\rightarrow$ La reconnaissance des établissements de formation postgraduée

\section{Enquête sur la qualité de la formation} postgraduée: les médecins-assistants évaluent leurs établissements de formation postgraduée

Avant de réaliser l'enquête, nous devons recenser le nombre de postes de formation postgraduée dans les 1500 établissements reconnus. Ce travail est également effectué au moyen d'une application électronique qui relève et évalue automatiquement sous forme statistique les données transmises par les médecins-chefs.

www.fmh.ch/awf $\rightarrow$ Pour les assistants/médecins en formation postgraduée $\rightarrow$ Enquête sur la qualité de la formation postgraduée

Registre des médecins - liste officielle de tous les médecins établis en Suisse De par la loi, la FMH est tenue d'élaborer une liste de tous les détenteurs d'un diplôme de médecin ou d'un titre postgrade. Le registre des médecins est l'unique répertoire contenant des données mises continuellement à jour. Pour y trouver les informations souhaitées, il suffit d'indiquer les qualifications professionnelles conformément à la RFP comme critère de recherche.

www.fmh.ch/awf $\rightarrow$ Divers $\rightarrow$ Registre des médecins

\section{Réglementation pour la formation continue (RFC)}

Les sociétés de discipline médicale, dont la compétence a été nettement renforcée par la nouvelle Réglementation pour la formation continue, sont désormais l'unique organe réglementant et mettant en œuvre la formation continue dans leur domaine spécifique. La FMH et les sociétés cantonales de médecine peuvent aussi attribuer des crédits dans ce domaine, mais uniquement pour des sessions non spécifiques portant sur des questions éthiques, de politique professionnelle, de santé ou de gestion. La plupart des sociétés ont adapté leurs programmes de formation continue en conséquence durant l'année écoulée.

$\mathrm{La}$ «formation continue pour le maintien des droits acquis» exigée dans le cadre du TARMED a été source d'inquiétudes. Durant l'exercice sous revue, le Comité central a approuvé un concept approprié réduisant les implications bureaucratiques à un minimum. Les principes de la responsabilité propre et de l'auto-déclaration ont été acceptés par les assureurs. 
Le Comité central a en outre approuvé seize programmes de formation continue.

www.fmh.ch/awf $\rightarrow$ Formation continue

\section{Caractère obligatoire de l'examen de spécialiste}

Durant l'exercice écoulé et après évaluation par le groupe d'experts pour l'examen de spécialiste (Prof. G. Stalder, Prof. U. Althaus et Mme le Dr $S$. Stöhr), la réussite a été confirmée comme étant obligatoire pour trois examens de plus, soit un de spécialiste et deux de formation approfondie. Actuellement, presque toutes les sociétés ont introduit le caractère obligatoire de l'examen de spécialiste. Il en va autrement des formations approfondies, dont les examens, dans bien des cas, doivent encore être soumis au groupe d'experts pour validation.

www.fmh.ch/awf $\rightarrow$ Pour les assistants/les médecins en formation postgraduée $\rightarrow$ Examens de spécialiste

L'intérêt des médecins étrangers à venir s'établir en Suisse ou du moins à y accomplir une formation postgraduée demeure très élevé, même si le nombre des demandes a reculé d'env. 25\% par rapport à l'exercice précédent.

Le peuple suisse ayant accepté d'étendre l'accord de libre passage aux 10 nouveaux pays de l'UE, nous avons commencé à revoir l'aide-mémoire FMH/OFSP. Des adaptations doivent être faites en fonction des nouveaux pays de l'UE, mais aussi des autorisations relevant du droit des étrangers et du blocage de l'accès à la pratique privée. Il est intéressant de relever l'évolution concernant l'immigration. En 2002 et 2003, le nombre de médecins étrangers immigrés était resté constant (574 et 755). Après la forte aug-

Barbara Linder

\section{Figure 8}

Diplômes de médecins et titres de formation postgraduée reconnus.

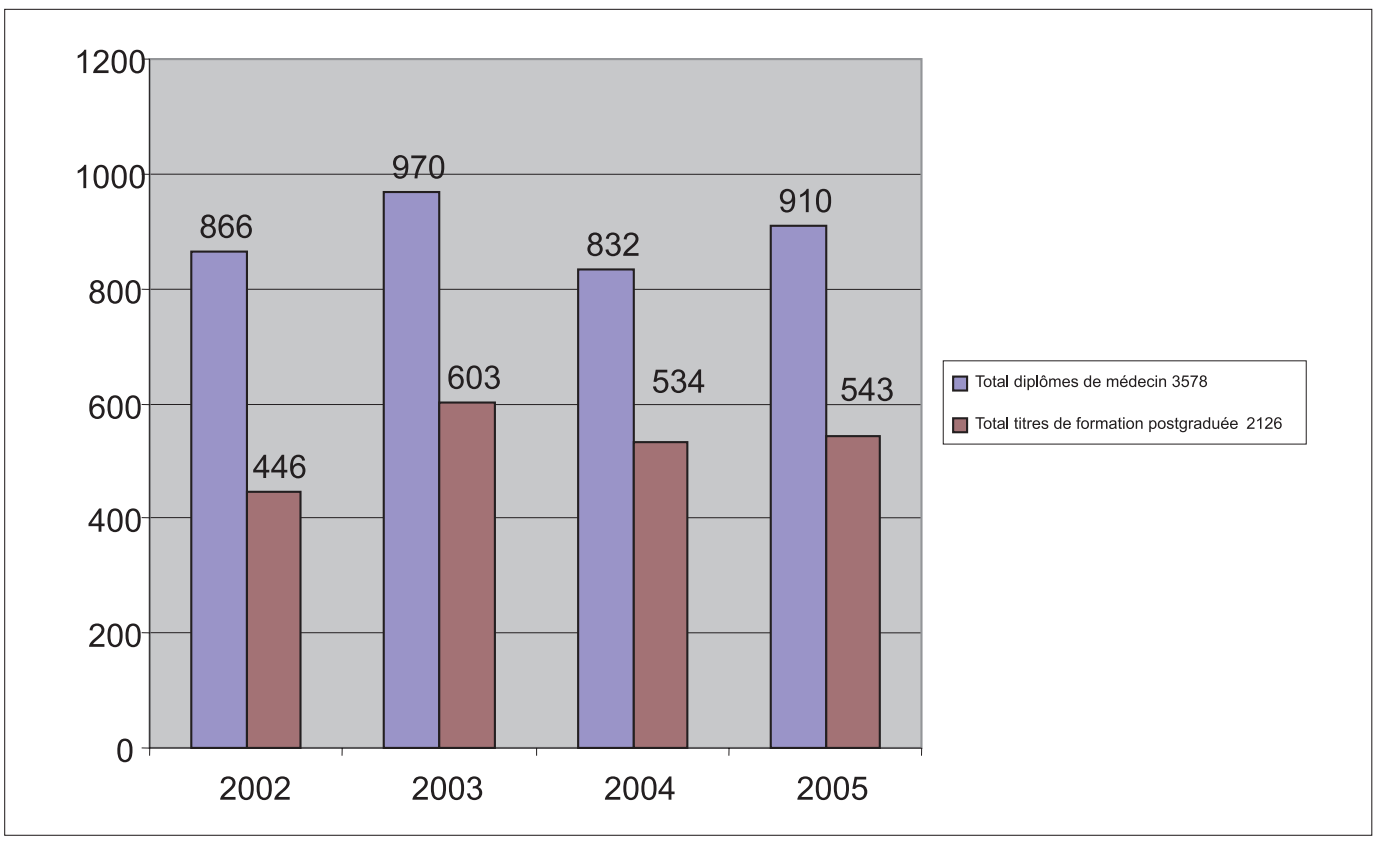


mentation enregistrée en 2004 (993), un léger recul s'est produit en 2005 (854).

www.fmh.ch/awf $\rightarrow$ Europe / Accords bilatéraux

\section{Office de conciliation pour les médecins- assistants et les chefs de clinique}

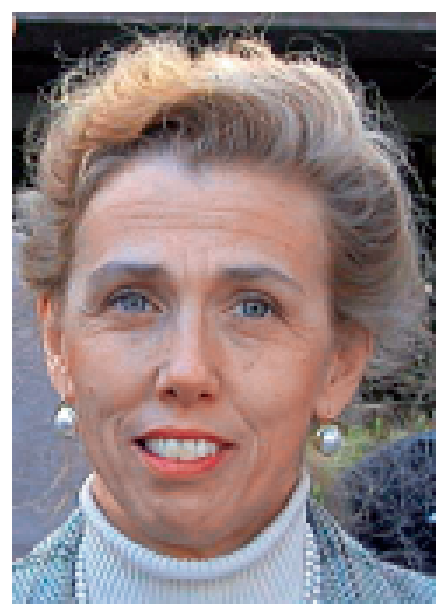

Dania Ischi, lic. en droit

Si des difficultés se présentent entre des candidats et des formateurs au cours de la formation postgraduée, la RFP permet aux deux parties de faire appel, dans différents domaines, à l'office de conciliation de la FMH selon l'art. 20, 4e al. RFP. Cette offre rencontre un large écho. Outre des questions relevant du droit du travail, les divergences portent principalement sur les certificats FMH et les protocoles d'évaluation. Nous constatons de plus en plus que, malgré la validation de la période de formation postgraduée accomplie, l'estimation des prestations dans le protocole d'évaluation ne correspond souvent pas à celle des candidats. La plupart du temps, les parties impliquées n'ont pas mené d'entretien d'évaluation intermédiaire, bien qu'un état des lieux pendant la période de formation soit vivement recommandé et qu'il soit de première importance dans cette situation. Un tel entretien permet d'éviter à l'avance non seulement les préjugés réciproques mais également, si possible, les surprises négatives et les déceptions à la fin de la période. De cette façon, on a plus de temps à disposition pour clarifier la situation lorsque les différends reposent sur de fausses suppositions ou un malentendu. Malheureusement une médiation n'est souvent demandée que lorsque le dialogue entre les parties n'est plus possible. En effectuant des négociations par étapes, nous tentons alors de parvenir à une solution acceptable par toutes les parties. Le taux élevé de réussite récompense nos efforts souvent dévoreurs de temps.

\section{Commissions d'opposition}

Commission d'opposition pour les titres de formation postgraduée (CO TFP)

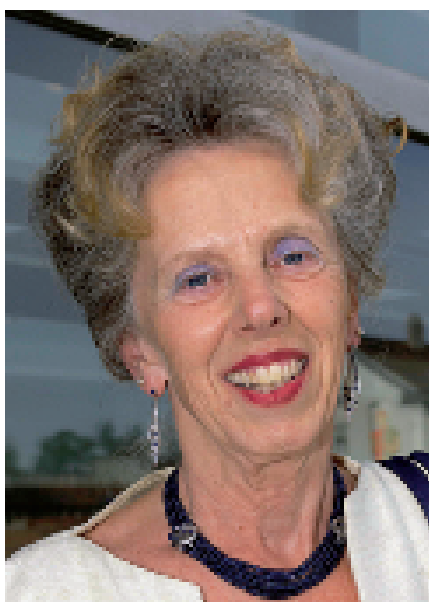

Dr Ursula Steiner-König (présidente)

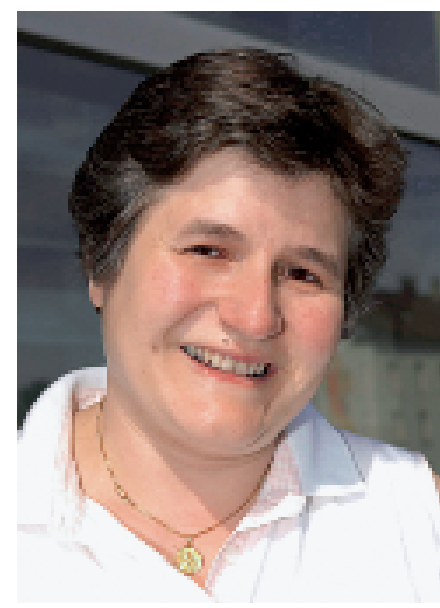

Dr Susanna Stöhr

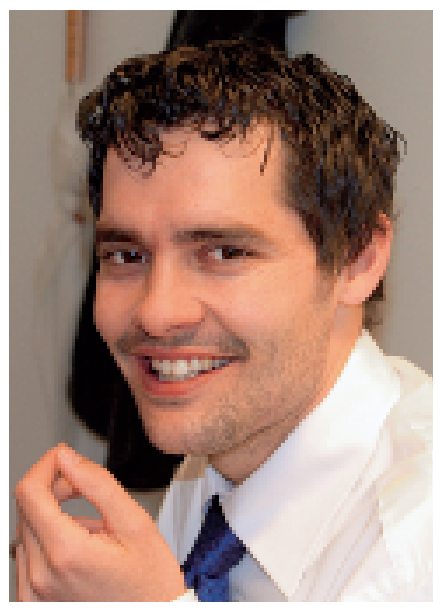

Simon Stettler, avocat

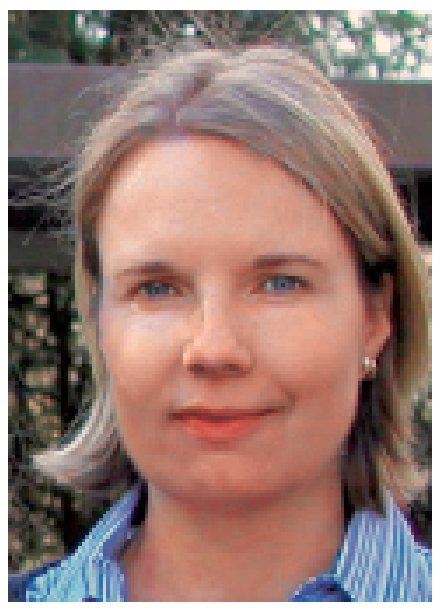

Nathalie Favre, lic. en droit 


\section{Commission d'opposition pour les établissements de formation postgraduée (CO EFP)}

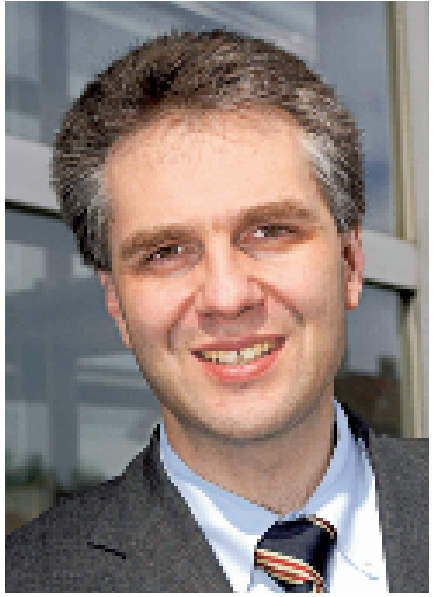

Dr Ludwig-Theodor Heuss (président)

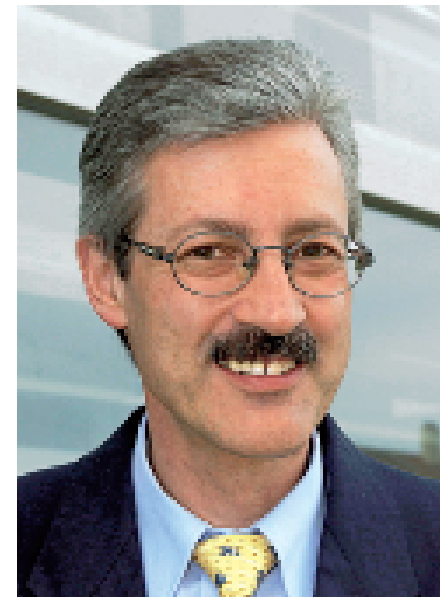

Dr Pierre-François Cuénoud

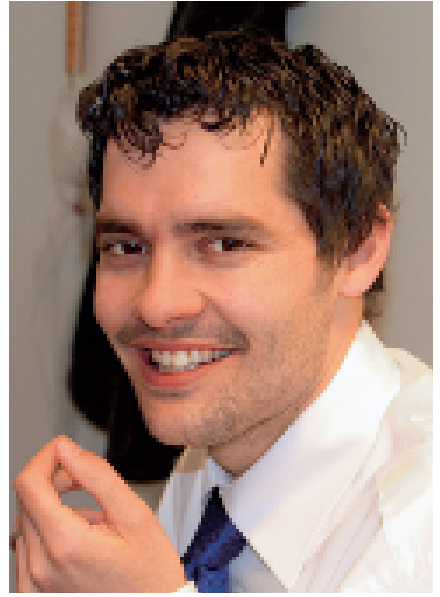

Simon Stettler, avocat

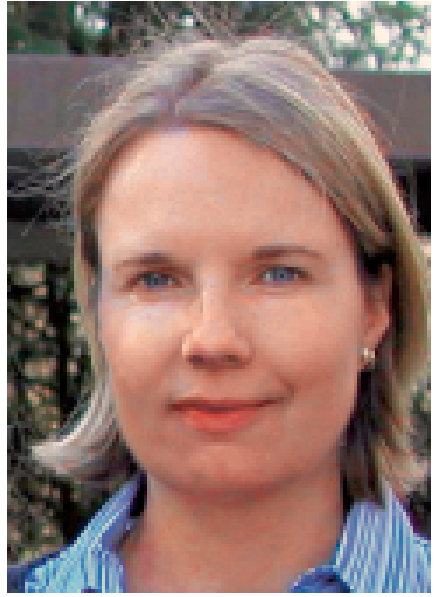

Nathalie Favre, lic. en droit
La Commission d'opposition pour les titres de formation postgraduée (CO TFP) se prononce sur les oppositions formées à l'encontre de décisions:

- de la Commission des titres (CT) portant notamment sur la validation des périodes de formation postgraduée accomplies en Suisse et à l'étranger, sur des demandes concernant la durée et la structure des curriculums de formation postgraduée et sur des demandes concernant l'attribution d'un titre de spécialiste;

- de responsables d'établissements de formation, relatives à la validation d'un certificat $\mathrm{FMH}$;
- de la Commission d'examen, concernant l'échec à un examen de spécialiste ou la nonadmission à un tel examen.

La Commission d'opposition pour les établissements de formation postgraduée (CO EFP) se prononce, quant à elle, sur les oppositions à l'encontre de décisions de la Commission des établissements de formation postgraduée (CEFP) relatives à la reconnaissance, à la classification et au changement de catégorie des établissements de formation.

Tableau 2

Oppositions concernant des titres de formation postgraduée en 2005.

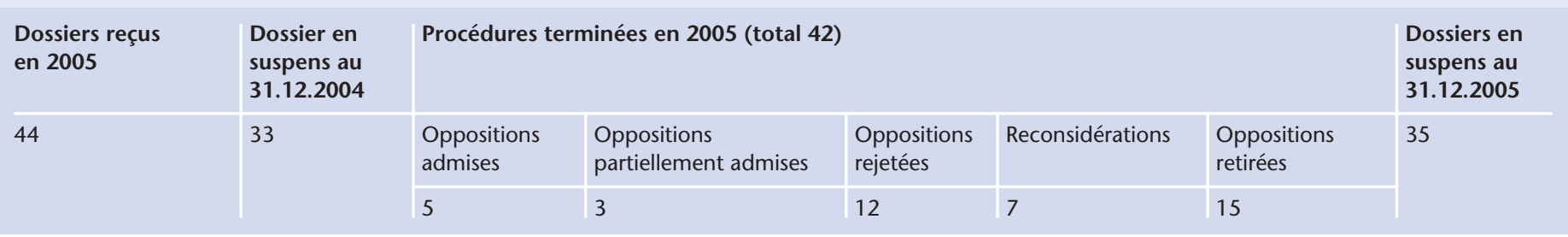

Tableau 3

Oppositions concernant des établissements de formation postgraduée 2005.

\begin{tabular}{|c|c|c|c|c|c|c|c|}
\hline Dossiers reçus & Dossier en & \multicolumn{5}{|c|}{ Procédures terminées en 2005 (total 42) } & \multirow{2}{*}{$\begin{array}{l}\text { Dossiers en } \\
\text { suspens au } \\
31.12 .2005 \\
7\end{array}$} \\
\hline \multirow[t]{2}{*}{5} & \multirow[t]{2}{*}{9} & $\begin{array}{l}\text { Oppositions } \\
\text { admises }\end{array}$ & $\begin{array}{l}\text { Oppositions } \\
\text { partiellement admises }\end{array}$ & $\begin{array}{l}\text { Oppositions } \\
\text { rejetées }\end{array}$ & Reconsidérations & $\begin{array}{l}\text { Oppositions } \\
\text { retirées }\end{array}$ & \\
\hline & & 0 & 0 & 0 & 3 & 4 & \\
\hline
\end{tabular}


Chaque décision des commissions d'opposition est prise par trois de leurs membres (deux membres du Comité central et un ou une juriste). En 2005, la CO TFP s'est réunie huit fois alors que la CO EFP n'a jamais siégé. Elle a en effet été en mesure de régler toutes les procédures d'opposition par voie de négociations (tab. 7).

Ces oppositions (42 au total) concernent les domaines suivants:

- Art. 23 RFP - Admission à l'examen de spécialiste (1)

- Art. 27 RFP - Examens de spécialiste insuffisants (12)

- Art. 38 RFP - Plans de formation postgraduée (15)

- Art. 46 RFP - Demandes de titre (14)

Les exigences liées aux catalogues d'opérations à effectuer ont fait l'objet de plusieurs procédures auprès de la CO TFP durant l'exercice écoulé. Pour les formations approfondies en chirurgie, il était possible jusqu'à fin 2005, sur la base de dispositions transitoires, d'obtenir le titre brigué uniquement en accomplissant le catalogue d'opérations exigé (jour de référence: 30 juin 2002). Ces oppositions n'ont pas toujours été faciles à juger. Les différents programmes de formation approfondie contiennent des dispositions plus au moins généreuses concernant les possibilités de compensation entre les opérations exigées. En outre, les commissions des titres des sociétés gérant une formation approfondie posent des conditions différentes quant à l'exhaustivité du catalogue des opérations à accomplir.

L'échec à un examen de spécialiste est un autre sujet auquel la CO TFP a été régulièrement confrontée. Ici, on observe régulièrement une divergence entre les attentes des candidats déçus et la compétence restreinte de la CO TFP en la matière. Par définition, la CO TFP n'est pas en mesure de réévaluer matériellement les prestations d'examen ni de donner un avis à la place des experts d'examen. Dans plusieurs cas, les opposants ont retiré leurs oppositions lorsqu'ils se sont rendu compte de cette situation. Un résultat insuffisant ne peut être annulé que s'il est matériellement indéfendable ou consécutif à une décision arbitraire. Même dans ce cas, les effets juridiques envisageables sont très restreints car le candidat peut repasser l'examen autant de fois qu'il le souhaite. Cette pratique de la CO TFP est en accord avec la jurisprudence du Tribunal fédéral. Le secrétariat de la FPPC a rédigé une notice indiquant la procédure à suivre concernant les oppositions à un examen de spécialiste insuffisant.

www.fmh.ch/awf $\rightarrow$ Pour les assistants/les médecins en formation postgraduée $\rightarrow$ Examens de spécialiste $\rightarrow$ Notice concernant les oppositions au refus d'un examen de spécialiste

Pour autant que la procédure d'opposition concerne une formation approfondie, la CO TFP rend une décision définitive. Quand il s'agit de titres de spécialiste fédéraux et d'établissements de formation postgraduée, la partie qui n'est pas d'accord avec la décision de la Commission d'opposition, peut faire recours auprès de la Commission fédérale de recours pour la formation de base et la formation postgrade des professions médicales (CRFPM). Dans un jugement de la CRFPM du 22 décembre 2005, lequel portait sur un litige relatif au refus d'octroi du titre de spécialiste en médecine générale à un candidat qui ne remplissait pas l'exigence de l'année à option obligatoire prévue par le programme de formation postgraduée et qui avait une longue formation postgraduée, la CRFPM a confirmé que la FMH n'avait pas abusé de la large autonomie qui lui est reconnue en la matière dans l'élaboration de ce programme. La CRFPM rappelle qu'un titre de spécialiste ne peut être délivré à un candidat que lorsque toutes les exigences du programme concerné sont remplies. Cela permet d'assurer l'organisation, le maintien et le contrôle de la qualité de la formation dispensée sur tout le territoire suisse. Cela permet également d'assurer une égalité de traitement de tous les candidats et satisfait à l'exigence de sécurité du droit. Enfin, la CRFPM souligne que, quelles que soient les qualités et l'expérience acquise du recourant, il ne se justifie pas de s'écarter du programme de formation accrédité.

Les décisions de la CRFPM peuvent être contestées devant le Tribunal fédéral. Durant la période sous revue, le Tribunal fédéral a traité deux recours. A chaque fois, il a confirmé la décision de la première instance et donc aussi celle de la Commission d'opposition de la FMH. Dans un cas, le Tribunal fédéral a constaté le caractère obligatoire de la liste des établissements de formation postgraduée reconnus par la FMH. L'autre procédure a porté sur la validation, pour le titre de spécialiste en médecine générale, d'études de psychologie accomplies avant l'acquisition du diplôme de médecin ainsi que sur la reconnaissance de plusieurs assistanats au cabinet médical sans certificat. 


\section{Autres domaines d'activité et projets du secrétariat FPPC}

\section{Révision de programmes de formation postgraduée}

Au cours de la période passée sous revue, le Comité central a adopté la révision de seize programmes de formation postgraduée.

La transformation de la formation approfondie en chirurgie de la main en un titre de spécialiste représentait une révision d'un genre particulier. Comme pour la neuropathologie, l'approbation accordée par la Chambre médicale suisse a toutefois été refusée par le FDI au motif que le nombre de titres de spécialiste existants est déjà très élevé. Il incombe maintenant à la FMH et aux sociétés de discipline concernées de déterminer la suite de la démarche.

www.fmh.ch/awf $\rightarrow$ Formation postgraduée $\rightarrow$ Pour les assistants / les médecins en formation postgraduée $\rightarrow$ Programmes de formation postgraduée et des attestations de formation complémentaire

\section{Loi fédérale sur les professions médicales universitaires (LPMéd)}

Après avoir franchi de nouveaux obstacles durant l'exercice écoulé, la loi sur les professions médicales (LPMéd) a été adoptée à l'unanimité par le Conseil national. Si le calendrier est respecté, la LPMéd remplacera en 2007/2008 l'actuelle loi sur l'exercice des professions médicales (LEPM) et conférera une nouvelle base légale à l'ensemble de la formation prégraduée, postgraduée et continue des cinq professions médicales universitaires. La question de savoir si, pour chaque profession médicale, une seule organisation ou plusieurs institutions doivent simultanément réglementer et décerner les titres de spécialiste reste un point litigieux. Le Conseil national s'est prononcé pour l'instant contre les idées de concurrence émises par le DFI et a repoussé ainsi la menace d'une scission de la formation postgraduée.

www.fmh.ch/awf $\rightarrow$ Loi sur les professions médicales (LPMéd)

www.bag.admin.ch/berufe/projektmed/f/index.htm

\section{Réforme des structures de la FMH/}

Projet de réforme de la CFPC

La réforme générale des structures de la FMH contient, entre autres, un projet de réforme de la CFPC qui vise à renforcer la CFPC en tant qu'organe dirigeant dans le domaine de la formation postgraduée et continue, et à en augmenter l'efficacité et l'efficience. Pour ce faire, le projet prévoit de doter le plénum de la CFPC de structures allégées et de compétences décisionnelles propres concernant la révision des programmes de formation postgraduée.

\section{Comptes 2005/Budget 2006-2011}

\section{Comptes 2005}

A l'instar de l'exercice précédent, les comptes 2005 bouclent avec un bénéfice de Fr. 744 000.-. Les charges comme les produits ont bénéficié d'une évolution plus favorable que celle budgétisée.

La majeure partie des charges est liée au personnel et aux compensations internes. Les frais de personnel se rapportent uniquement aux personnes engagées au secrétariat FPPC. Toute l'infrastructure, le travail des autres services et départements de la FMH ainsi que les organes de la FMH sont indemnisés par des compensations internes au prorata. Grâce à une comptabilité analytique tenue avec précision, le secrétariat de la FPPC peut chiffrer de manière exacte les coûts de chaque tâche et projet en particulier.

\begin{tabular}{|c|c|}
\hline $\begin{array}{l}\text { Enquête auprès des médecins-assistants } \\
\text { sur la qualité de la formation postgraduée }\end{array}$ & Fr. 177 000.- \\
\hline Accréditation de la FMH & Fr. $163000 .-$ \\
\hline Commissions d'opposition & Fr. $160000 .-$ \\
\hline $\begin{array}{l}\text { Domaine des établissements } \\
\text { de formation postgraduée }\end{array}$ & Fr. $113000 .-$ \\
\hline Domaine de la formation continue & Fr. $38000 .-$ \\
\hline $\begin{array}{l}\text { Contacts avec l'étranger (demandes } \\
\text { émanant de médecins étrangers/ } \\
\text { d'organisations étrangères) }\end{array}$ & Fr. $34000 .-$ \\
\hline $\begin{array}{l}\text { Attestations de formation complé- } \\
\text { mentaire (administration des données, } \\
\text { élaboration de programmes) }\end{array}$ & Fr. $26000 .-$ \\
\hline
\end{tabular}

\section{Budget 2006/2007}

La baisse persistante du nombre de titres décernés aura des effets directs sur le budget de ces prochaines années. Pour 2006, nous comptons encore sur des recettes d'env. 3,8 millions de francs. A long terme, la taxe de Fr. 4000.- pour l'acquisition d'un titre de spécialiste ne couvrira plus entièrement les charges de la FMH dans le domaine de la formation postgraduée et continue. Les réserves accumulées depuis 2002 permettent néanmoins de combler ce déficit pen- 
dant plusieurs années. La Chambre médicale s'est déjà prononcée en 2001 clairement en faveur d'un soutien de la formation postgraduée par le biais des cotisations générales, ce qui témoigne d'une solidarité vivante entre les générations. Etant donné l'état actuel de la fortune (au $1^{\text {er }}$ janvier 2006), nous disposons de moyens financiers suffisants pour mener à terme les projets d'assurance-qualité qui ont été lancés.
Tableau 5

Etat de la fortune FPPC au 1.1.2006

\begin{tabular}{ll} 
Pertes 2002 & Fr. $-999000 .-$ \\
\hline Contribution de solidarité 2002 & Fr. 600000 .- \\
\hline Bénéfice 2003 & Fr. 1704000 .- \\
\hline Contribution de solidarité 2003 & Fr. $1200000 .-$ \\
\hline Bénéfice 2004 & Fr. 740000 .- \\
\hline Bénéfice 2005 & Fr. $744000 .-$ \\
Solde & Fr. 3989000 .-
\end{tabular}

\section{Produits}

Titres de spécialiste (2005: 1039)

\section{Comptes 2005}

Formations approfondies (2005: 186)

Médecins praticiens (2005: 135)

Attestations d'équivalence (2005: 31)

Renseignements/Plans de formation

Oppositions

Divers (réimpression de diplômes)

Attestations de formation

complémentaire

Visites d'établissements de formation postgraduée

Reconnaissance de sessions de formation continue par la FMH

Projet OFSP (tâches déléguées par I'OFSP)

Autres produits

TOTAL des produits

\section{Charges}

Charges pour prestations de tiers

Indemnités CT

Indemnités CFPC

Indemnités Experts aux examens

de spécialiste

Indemnités CEFP

Indemnités Visites d'établissement

Frais de manifestation/Séances

3702000

174000

154000

31000

19000

6000

4000

1000

106000

4000

102000

1000

4303000

Comptes 2005
0

3857000

3731000

Budget 2006

Budget 2007

\begin{tabular}{|r|r|r|}
\hline 120000 & 150000 & 130000 \\
\hline 54000 & 45000 & 95000 \\
\hline 5000 & 5000 & 5000 \\
\hline 39000 & 30000 & 30000 \\
\hline 53000 & 150000 & 100000 \\
\hline 4000 & 13000 & 8000 \\
\hline
\end{tabular}




\section{Charges}

Charges pour prestations de tiers

Dépenses directes pour prestations de service (frais d'impression de diplômes)

Dépenses pour projets (accréditation, enquête auprès des assistants, formation postgraduée en matière de gestion)

Concepts de formation postgraduée/ Visites d'établissement (manuels)

\section{Comptes 2005}

Budget 2006

Budget 2007

Total des charges pour prestations de tiers

Frais de personnel

Rémunérations et salaires (2005:

2 membres CC, 1 administrateur, 13 collaboratrices)

\begin{tabular}{|c|c|c|c|}
\hline Assurances sociales & 256000 & 309000 & 299000 \\
\hline Autres frais de personnel & 39000 & 63000 & 53000 \\
\hline Prestations de tiers & 0 & 0 & 2000 \\
\hline Total des frais de personnel & 1660000 & 1872000 & 1804000 \\
\hline \multicolumn{4}{|l|}{ Autres frais d'exploitation } \\
\hline Entretien et réparations & 0 & 6000 & 2000 \\
\hline Véhicules et transport & 1000 & 1000 & 1000 \\
\hline Administration et informatique & 32000 & 195000 & 191000 \\
\hline Publicité & 1000 & 10000 & 0 \\
\hline Total des autres frais d'exploitation & 34000 & 212000 & 194000 \\
\hline \multicolumn{4}{|l|}{$\begin{array}{l}\text { Compensations internes } \\
\text { (prestations «achetées» à la FMH } \\
\text { par le secrétariat FPPC et vice-versa) }\end{array}$} \\
\hline $\begin{array}{l}\text { A) Compensations internes } \\
\text { selon la comptabilité analytique }\end{array}$ & & & \\
\hline $\begin{array}{l}\text { TIC (technologies d'information } \\
\text { et de communication) }\end{array}$ & 23000 & 20000 & 30000 \\
\hline Traduction & 102000 & 131000 & 117000 \\
\hline Assistance informatique & 41000 & 55000 & 55000 \\
\hline Service juridique & 149000 & 162000 & 125000 \\
\hline Autres & 25000 & 24000 & 15000 \\
\hline Moins les prestations fournies à la FMH & -230000 & -216000 & -155000 \\
\hline
\end{tabular}


B) Compensations internes selon forfaits

\begin{tabular}{|c|c|c|c|}
\hline Location & 65000 & 65000 & 66000 \\
\hline $\begin{array}{l}\text { Matériel de bureau/Impression/ } \\
\text { Frais de port }\end{array}$ & 75000 & 75000 & 75000 \\
\hline Téléphone, comptabilité & 165000 & 165000 & 165000 \\
\hline Informatique & 250000 & 250000 & 250000 \\
\hline $\begin{array}{l}\text { Chambre médicale, Conférence } \\
\text { des présidents }\end{array}$ & 42000 & 42000 & 42000 \\
\hline Comité Central & 188000 & 188000 & 188000 \\
\hline Administration du Secrétariat général & 120000 & 120000 & 120000 \\
\hline Registre des médecins & 163000 & 163000 & 150000 \\
\hline Total des compensations internes & 1178000 & 1244000 & 1243000 \\
\hline \multirow[t]{2}{*}{ TOTAL des charges } & 3559000 & 4091000 & 3954000 \\
\hline & Comptes 2005 & Budget 2006 & Budget 2007 \\
\hline Charges & 3559000 & 4091000 & 3954000 \\
\hline Produits & 4303000 & 3857000 & 3731000 \\
\hline Gain (+)/Perte (-) d'exploitation & 744000 & -234000 & -223000 \\
\hline Restitutions de cotisations & -1180000 & -1120000 & -1553000 \\
\hline Compensation des restitutions & 1180000 & 1120000 & 1553000 \\
\hline RÉSULTAT & 744000 & -234000 & -223000 \\
\hline
\end{tabular}

\section{Perspectives et objectifs}

Pour l'année en cours, notre objectif principal est de mener à bon port la loi sur les professions médicales actuellement débattue au Parlement, de manière à ce que la compétence de gérer l'ensemble des titres de spécialiste ne revienne, à l'avenir également, qu'à UNE seule et même organisation. La cohérence des programmes de formation postgraduée ne pourra être garantie qu'à cette condition. Plusieurs milieux souhaitent que la formation postgraduée ne soit plus réglée par la seule $\mathrm{FMH}$, mais conjointement par toutes les organisations importantes actives dans le secteur de la formation postgraduée médicale. La FMH est encore en train de définir sa position à ce sujet et lorsque ce processus sera terminé, il revêtira une grande importance pour l'orientation stratégique du corps médical. Une Commission pour la formation postgraduée et continue largement indépendante et munie de compétences propres (CFPC), comprenant des représentants des groupes concernés les plus importants, s'inscrit déjà dans ledit processus. Nous avons également pour objectif important en 2006 de mener à terme le projet de réforme de la CFPC, puis de procéder à sa mise en œuvre. 